\title{
The Software Language Extension Problem
}

\author{
Manuel Leduc ${ }^{1} \cdot$ Thomas Degueule $^{2} \cdot$ Eric Van $\mathrm{Wyk}^{3} \cdot$ Benoit Combemale $^{4}$
}

Received: 5 September 2019 / Revised: 3 November 2019 / Accepted: 4 December 2019

(c) Springer-Verlag GmbH Germany, part of Springer Nature 2019

\begin{abstract}
The problem of software language extension and composition drives much of the research in Software Language Engineering (SLE). Although various solutions have already been proposed, there is still little understanding of the specific ins and outs of this problem, which hinders the comparison and evaluation of existing solutionsIn this SoSyM Expert Voice, we introduce the Language Extension Problem as a way to better qualify the scope of the challenges related to language extension and compositionThe formulation of the problem is similar to the seminal Expression Problem introduced by Wadler in the late 1990s and lifts it from the extensibility of single constructs to the extensibility of groups of constructs, i.e., software languages. We provide a comprehensive definition of the actual constraints when considering language extension and believe the Language Extension Problem will drive future research in SLE, the same way the original Expression Problem helped to understand the strengths and weaknesses of programming languages and drove much research in programming languages.
\end{abstract}

Keywords Domain-specific language $\cdot$ Extension $\cdot$ Composition $\cdot$ Expression problem

\section{Introduction}

With the advent of language workbenches, the problem of modular language extension has garnered considerable interest from the research community in the past decade. This problem informally. refers to the capability of extending the syntax and semantics of an existing language while reusing its specification (e.g., grammars, semantic inference rules) and implementation (e.g., parsers, interpreters). Various authors have attempted to formalize this problem (e.g., [5]), but the lack of a. clear definition makes it hard to

Communicated by Bernhard Rumpe.

Benoit Combemale

benoit.combemale@irit.fr

Manuel Leduc

manuel.leduc@irisa.fr

Thomas Degueule

degueule@cwi.nl

Eric Van Wyk

evw@umn.edu

1 IRISA, CNRS, Univ Rennes, Inria, Rennes, France

2 CWI, Amsterdam, The Netherlands

3 University of Minnesota, Minneapolis, USA

4 University of Toulouse and Inria, Toulouse, France evaluate and compare the strengths and weaknesses of existing solutions w.r.t. a common, well-defined framework. This paper is an attempt to define language extensibility in the form of a well-defined problem.

\section{From the Expression Problem to the Language Extension Problem}

Philip Wadler coined the term "Expression Problem" to name a well-known problem in the programming languages community, and this name has been in common use for more than two decades [21]. As Oliveira and Cook put it [15]:

The "expression problem" (EP) is now a classical problem in programming languages. It refers to the difficulty of writing data abstractions that can be easily extended with both new operations and new data variants.

Over time, the EP has made it possible to structure the discussions around the capabilities of different programming paradigms and languages regarding datatypes extensibility using a common set of constraints that candidate solutions should address. There are different variations in the EP, but its canonical definition includes the following constraints [22]:

Extensibility in both dimensions It should be possible to add new data variants and adapt existing operations 
accordingly. Furthermore, it should be possible to introduce new operations.

Strong static-type safety It should be impossible to apply an operation to a data variant that the operation cannot handle.

No modification or duplication Existing code should neither be modified nor duplicated.

Separate compilation Compiling datatype extensions or adding new operations should not encompass re-typechecking the original datatype or existing operations.

Independent extensibility It should be possible to combine independently developed extensions so that they can be used jointly.

There is a striking parallel between the problem of modular language extension and the Expression Problem. As a matter of fact, most approaches to modular language extension end up discussing and addressing the EP in some way $[8,12]$. However, in the context of Software Language Engineering (SLE), "data variants" are groups of syntactic categories and their constructors and "operations" over these data variants define their semantics. Due to the ambiguity in the name Expression Problem, in which "expression" may refer to a language of expressions, one might naively think that the EP and the problem of modular language extension are equivalent.

In this paper, we demonstrate that instantiating the EP in the context of SLE requires to reformulate and refine the existing constraints of the EP and to introduce new ones, leading to a new problem: the Language Extension Problem (LEP). While the EP is merely a programming problem concerning programmers and focusing on the extensibility of a single datatype, the LEP is a Software Language Engineering (SLE) problem concerning language engineers and focusing on the extensibility of languages (i.e., group of datatypes representing the language constructs). The LEP must also account for engineering practices that are specific to software languages such as the use of language workbenches, the duality of language specifications and implementations, and the specificities of syntax definition. As extending a group of datatypes entails extending the datatypes it is composed of, in many cases solving the LEP (in the large) entails solving the EP (in the small). We identify what is the meaning of the two extension axes in this context and what is the set of constraints that must be used to assess the success of a given solution. Naturally, many partial solutions to the LEP already exist in the literature, scattered from programming language theory (e.g., modular visitor components [14], Revisitors [12], Recaf [1]), to language workbenches (e.g., Rascal [10], Melange [3], Silver [9,18]). We purposely limit ourselves to the definition of the LEP and leave to future work the positioning of existing solutions w.r.t. the constraints we list.

\section{The language expression problem}

The Expression Problem has been initially introduced in the context of datatype extension and composition and hence presented in terms of datatypes and functions over the datatypes. Conversely, the Language Extension Problem focuses on language extension and composition, presented in terms of, respectively, syntax in the form of multiple syntactic categories and constructors for each category, and semantics over that syntax. In the following, we introduce the Language Extension Problem by paraphrasing the original definition of the Expression Problem by Wadler [21], but lifting the vocabulary from datatypes to languages.

The Language Extension Problem (LEP) is a new name for an old problem. The goal is to define a family of languages, where one can add a new language to the family by adding new syntax (i.e., new constructors for existing syntactic categories as well as new categories) and also new semantics over existing and new syntax, while conforming to constraints similar to those in the Expression Problem but specialized to language extension.

As an example, consider a language family regarding state machines which starts from a core language over simple finite state machines with a simple pretty-printing semantics and constructs a new language by adding syntax to specify hierarchical state machines and a new semantics to evaluate state machines given an input sequence.

According to this characterization of the LEP, we now review the constraints initially identified in the EP and express them in the context of SLE for the LEP:

Extensibility in both dimensions It should be possible to extend the syntax and adapt existing semantics accordingly. Furthermore, it should be possible to introduce new semantics on top of the existing syntax.

Strong static-type safety All semantics should be defined for all syntax.

No modification or duplication Existing language specifications and implementations should neither be modified nor duplicated.

Separate compilation Compiling a new language (e.g., syntactic extension or new semantics) should not encompass re-compiling the original syntax or semantics.

Independent extensibility It should be possible to combine and used jointly language extensions (syntax or semantics) independently developed.

Moreover, the distinction between the specification and the implementation of new engineered languages raises a new concern regarding automatic composition [8]. Indeed, "glue 


\section{(a)}

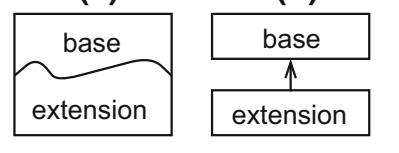

(c)

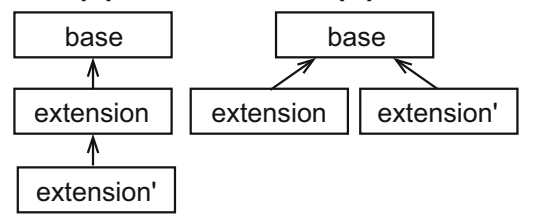

extension' (e)

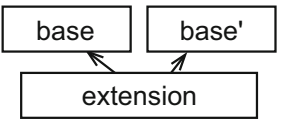

Fig. 1 Approaches for language extension, applicable at the specification and implementation levels. a mixes up the extension into the base language, while b-e keep them separated and use explicit operators (e.g., references, static/dynamic introduction) or glue code

code," i.e., code dedicated solely to the interconnection of extensions, must be limited or avoided from the user's point of view to compose a collection of language extensions.

\section{The LEP in practice}

Numerous approaches have been explored in the past decade to address specific scenarios of languages extension, either at the specification level (e.g., $[2,4,6,9,11,13,17,19,20,22]$ ) or at the implementation level (e.g., [7,16,22,23]). The specification level is based on metalanguages that provide the relevant abstractions, often with limited and domain-specific expressiveness. The specification level is then turned into an implementation, thanks to compilation or interpretation, often by targeting general-purpose programming languages and following language implementation patterns specific to each approach.

While all those approaches are heterogeneous and conceptually operate at different levels, they share common extension mechanisms which are summarized in the five approaches depicted in 1 [5].

Complying exhaustively to the identified constraints is extremely challenging, and trade-offs must be considered for a given context. We present a selection of scenarios illustrating such trade-offs. First, the constraint of separate compilation usually impacts other non-functional properties such as performance, readability, and accidental complexity (e.g., large and complex glue code, unclear modules dependencies). Consequently, it can be worthwhile to relax the separate compilation constraint in order to comply with other non-functional properties. Second, various actors can be responsible for language extension. They each come with very different skills, ranging from SLE experts with a deep understanding of languages and language workbenches internals, to end users with minimal knowledge of software development. While the former is capable of performing composition using complex handwritten glue specifications, the latter will typically require fully automatic composition approaches. Finally, the boundaries of a language family are important to consider. Two statuses can be considered, closed (i.e., all its languages are known) and open (i.e., new languages can be added organically). Indeed, in the context of closed families, the compatibility of the extensions can be checked in advance and conforming to the type-safe constraints is not an issue. On the contrary, in the context of open language families, restrictive-type systems can lead to difficulty or impossibility to extend languages in unanticipated contexts.

The constraints of the Language Extension Problem define a framework for comparing language extension approaches. It is worth noting that conforming or not to some of the constraints is often the consequence of interesting language design choices, relative to some specific scenarios.

\section{Conclusion}

In this column, we describe the Language Extension Problem, a lift of the Expression Problem at the language level. We lift the constraints drawn from the Expression Problem to the context of software language engineering, and introduced an additional constraint specific to this context. Through the Language Extension Problem, we hope to provide a framework to reason on language extension and its challenges and help the comparison of existing and future SLE contributions.

\section{References}

1. Biboudis, A., Inostroza, P., Storm, T.V.: Recaf: Java dialects as libraries. ACM SIGPLAN Notices 52(3), 2-13 (2017)

2. Butting, A., Eikermann, R., Kautz, O., Rumpe, B., Wortmann, A.: Systematic composition of independent language features. J. Syst. Softw. 152, 50-69 (2019). https://doi.org/10.1016/j.jss.2019.02. 026

3. Degueule, T., Combemale, B., Blouin, A., Barais, O., Jézéquel, J.M.: Melange: A meta-language for modular and reusable development of dsls. In: Proceedings of the 2015 ACM SIGPLAN International Conference on Software Language Engineering, pp. 25-36. ACM (2015)

4. Degueule, T., Combemale, B., Blouin, A., Barais, O., Jézéquel, J.M.: Safe model polymorphism for flexible modeling. Comput. Lang. Syst. Struct. 49, 30 (2016). https://doi.org/10.1016/j.cl.2016. 09.001 
5. Erdweg, S., Giarrusso, P.G., Rendel, T.: Language composition untangled. In: A. Sloane, S. Andova (eds.) International Workshop on Language Descriptions, Tools, and Applications, LDTA '12, Tallinn, Estonia, March 31-April 1, 2012, p. 7. ACM (2012). 10.1145/2427048.2427055

6. Erdweg, S., Rendel, T., Kastner, C., Ostermann, K.: SugarJ: Library-based syntactic language extensibility. In: Proceedings of ACM SIGPLAN Conference on Object Oriented Programming, Systems, Languags, and Applications (OOPSLA), pp. 391-406. ACM (2011). 10.1145/2048066.2048099

7. Hills, M., Klint, P., van der Storm, T., Vinju, J.J.: A case of visitor versus interpreter pattern. In: J. Bishop, A. Vallecillo (eds.) Objects, Models, Components, Patterns: 49th International Conference, TOOLS 2011, Zurich, Switzerland, June 28-30, 2011. Proceedings, Lecture Notes in Computer Science, vol. 6705, pp. 228-243. Springer (2011). 10.1007/978-3-642-21952-8_17

8. Kaminski, T., Kramer, L., Carlson, T., Van Wyk, E.: Reliable and automatic composition of language extensions to $\mathrm{C}$ : The ablec extensible language framework. PACMPL 1(OOPSLA), 98:198:29 (2017). 10.1145/3138224

9. Kaminski, T., Van Wyk, E.: Modular well-definedness analysis for attribute grammars. In: Proceedings of the 5th International Conference on Software Language Engineering (SLE), Lecture Notes in Computer Science, vol. 7745, pp. 352-371. Springer, Berlin(2012). 10.1007/978-3-642-36089-3_20

10. Klint, P., Van der Storm, T., Vinju, J.: Rascal: A domain specific language for source code analysis and manipulation. In: Source Code Analysis and Manipulation, 2009. SCAM'09. Ninth IEEE International Working Conference on, pp. 168-177. IEEE (2009)

11. Leduc, M., Degueule, T., Combemale, B.: Modular language composition for the masses. In: D. Pearce, T. Mayerhofer, F. Steimann (eds.) Proceedings of the 11th ACM SIGPLAN International Conference on Software Language Engineering, SLE 2018, Boston, MA, USA, Nov 05-06, 2018, pp. 47-59. ACM (2018). 10.1145/3276604.3276622

12. Leduc, M., Degueule, T., Combemale, B., Van der Storm, T., Barais, O.: Revisiting visitors for modular extension of executable dsmls. In: 2017 ACM/IEEE 20th International Conference on Model Driven Engineering Languages and Systems (MODELS), pp. 112122. IEEE (2017)

13. Mernik, M.: An object-oriented approach to language compositions for software language engineering. J. Syst. Softw. 86(9), 24512464 (2013). https://doi.org/10.1016/j.jss.2013.04.087

14. Oliveira, B.C.d.S.: Modular visitor components. In: European Conference on Object-Oriented Programming, pp. 269-293. Springer, Berlin (2009)

15. d. S. Oliveira, B.C., Cook, W.R.: Extensibility for the masses - practical extensibility with object algebras. In: ECOOP 2012Object-Oriented Programming: 26th European Conference, Beijing, China, June 11-16, 2012. Proceedings, pp. 2-27 (2012)

16. Torgersen, M.: The expression problem revisited. In: Odersky, M. (ed.) ECOOP 2004-Object-Oriented Programming, pp. 123-146. Springer, Berlin (2004)

17. Vacchi, E., Cazzola, W.: Neverlang: A framework for featureoriented language development. Comput. Lang. Syst. Struct. 43, 1-40 (2015). https://doi.org/10.1016/j.cl.2015.02.001

18. Van Wyk, E., Bodin, D., Gao, J., Krishnan, L.: Silver: an extensible attribute grammar system. Sci. Comput. Program. 75(1-2), 39-54 (2010). https://doi.org/10.1016/j.scico.2009.07.004

19. Voelter, M.: Language and IDE modularization and composition with MPS. In: Generative and Transformational Techniques in Software Engineering IV, International Summer School, GTTSE 2011, Braga, Portugal July 3-9, 2011. Revised Papers, pp. 383430 (2011). 10.1007/978-3-642-35992-7_11
20. Wachsmuth, G., Konat, G.D.P., Visser, E.: Language design with the spoofax language workbench. IEEE Softw. 31(5), 35-43 (2014). https://doi.org/10.1109/MS.2014.100

21. Wadler, P.: The expression problem (1998). http://homepages.inf. ed.ac.uk/wadler/papers/expression/expression.txt. Posted on the Java Genericity Mailing List, 12 Nov 1998

22. Zenger, M., Odersky, M.: Independently extensible solutions to the expression problem. Tech. rep. (2004)

23. Zhang, W., Oliveira, BCdS: EVF: an extensible and expressive visitor framework for programming language reuse (artifact). DARTS 3(2), 10:1-10:2 (2017)

Publisher's Note Springer Nature remains neutral with regard to jurisdictional claims in published maps and institutional affiliations.

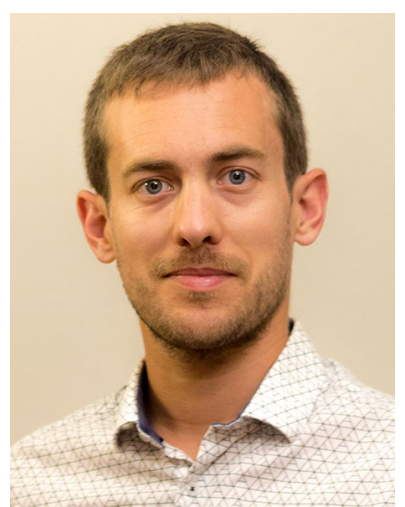

Manuel Leduc is currently a $\mathrm{Ph} . \mathrm{D}$. candidate at the University of Rennes 1 in France. His research interests include on Model-Driven Engineering and Software Language Engineering. Before this, he worked in the industry for 4 years as a software engineer and software system architect.

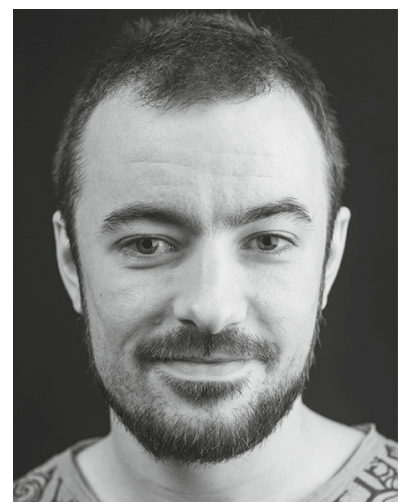

Thomas Degueule is a researcher in the Software Analysis and Transformation Group (SWAT) at Centrum Wiskunde \& Informatica (CWI), Amsterdam. He received his Ph.D. degree from Inria and the University of Rennes 1. His research pertains to software engineering in general and focuses on the design, implementation, and use of software languages to assist developers and domain experts in the engineering of complex software systems. 


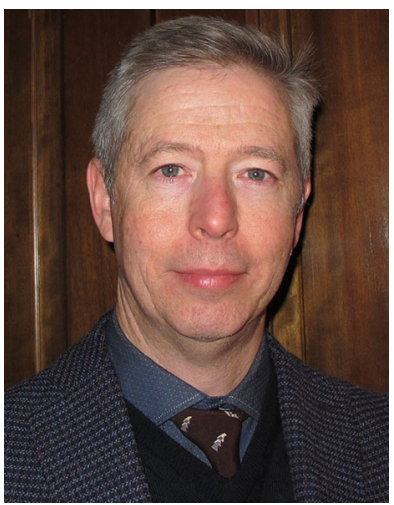

Eric Van Wyk is an Associate Professor in the Department of Computer Science and Engineering at the University of Minnesota. He earned a Ph.D. at the University of Iowa and was a post-doctoral researcher at the Oxford University Computing Laboratory. His research focuses on tools and techniques for specifying extensible programming languages. He was the recipient of a National Science Foundation CAREER Award in 2004 and the College of Science and Engineering Charles E.

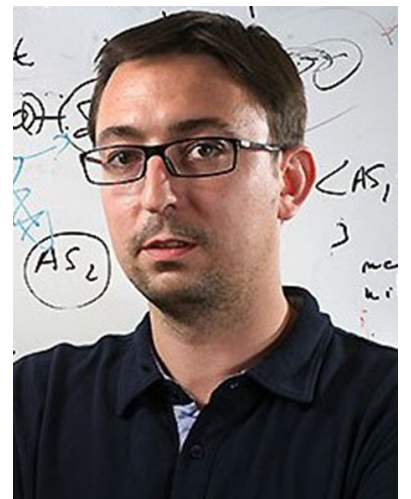

Benoit Combemale is Full Professor of Software Engineering at the University of Toulouse, and Research Scientist at Inria. His research interests are in the field of software engineering, including Model-Driven Engineering, Software Language Engineering and Validation \& Verification; mostly in the context of (smart) CyberPhysical Systems and Internet of Things. He is also teaching worldwide in various engineering schools and universities. More information at http://combemale.fr

Bowers Faculty Teaching Award in 2017. 